\title{
Die Constitution des Ornithins und des Lysins. Zugleich ein Beitrag zur Chemie der Eiweissfäulniss.*)
}

Von

Dr. phil. et med. Alexander Ellinger, Privatdocent und Assistent des Instituts.

(Aus dem Universitäts-Laboratorium für medicinische Chemie in Königsberg i. Pr.)

(Der Redaction zugegangen am 18. März 1900.)

Seitdem Drechsel1) die Entdeckung gemacht hat, dass bei der hydrolytischen Spaltung von Eiweisskörpern nicht nur die bekannten Monoamidosäuren als Zersetzungsprodukte auftreten, sondern auch Substanzen von ausgesprochen basischem Charakter, sind diese Basen ein bevorzugtes Gebiet der physiologisch-chemischen Forschung geworden. Durch wesentliche Vervollkommnung der Methodik, welche wir vorzugsweise Hedin ${ }^{2}$ ) und A. $\mathrm{Kossel}^{3}$ ) verdanken, gelang es, in allen daraufhin untersuchten Eiweisskörpern die drei basischen Spaltungsprodukte Histidin, Arginin und Lysin aufzufinden und diese drei Substanzen als wesentliche Zerfallsprodukte der Protamine, welche Kossel als die einfachsten Eiweisskörper anspricht, nachzuweisen. Nach diesen Befunden fand auch die Entstehung des Arginins in Lupinen- und Kürbiskeimlingen,

*) Eine kurze Veröffentlichung über die hauptsächlichsten Resultate dieser Arbeit ist bereits in den Berichten der chemischen Gesellschaft 31,3183 und 32,3542 erschienen.

1) Ber. d. math.-phys. Klasse der Kgl. Sächs. Gesellsch. d. Wiss. 1892 S. 116.

2) Diese Ztschr. Bd. XXI, S. 155.

3) Diese Ztschr. Bd. XXV, S. 165. 
worin es von E. Schulze uud Steiger ${ }^{1}$ ) entdeckt worden war, seine Erklärung.

Von den drei genannten Basen ist die Molekularformel durch zahlreiche Analysen wohl krystallisirender Salze, theilweise auch durch Molekulargewichtbestimmungen festgestellt; aber allein beim Arginin ist es bisher gelungen, durch Spaltungsreactionen einen Einblick in die Constitution $z u$ erlangen. Schulze und $\mathrm{W}$ interstein ${ }^{2}$ ) nämlich fanden, dass das Arginin beim Erhitzen mit Barytwasser in Harnstoff und Ornithin zerfällt. Sie sprachen daraufhin das Arginin als ein Guanidinderivat, ähnlich dem Kreatin an, in welchem eine Amidogruppe durch den Ornithinrest ersetzt ist. In neuster Zeit ist den beiden Forschern ${ }^{3}$ ) auch die Bestätigung ihrer Anschauung auf synthetischem Wege geglückt. Sie erhielten, analog der Kreatinsynthese aus Cyanamid und Methylglycocoll, das Arginin aus Cyanamid und Ornithin. Die interessanten Befunde von Schulze und Winterstein, dass das Ornithin ein Spaltungsprodukt aller Eiweisskörper sei, legten diesem Körper wieder ein erhöhtes Interesse bei.

Das Ornithin ist bekanntlich von Jaffé ${ }^{4}$ ) aus der Ornithursäure durch Spaltung mit Salzsäure in Form seines salzsauren Salzes erhalten werden. Die Ornithursäure, das Dibenzoylderivat des Ornithins, wurde im Harn von Hühnern nach Fütterung mit Benzoësäure aufgefunden.

Auf Grund der Analyse einer Reihe von Salzen sprach Jaffé das Ornithin als eine Diamidovaleriansäure an. Diamidosäuren der Fettreihe waren damals weder synthetisch dargestellt noch im Thier- oder Pflanzenkörper aufgefunden. Erst 15 Jahre nach der Entdeckung des Ornithins wurde eine Substanz gefunden, die nach ihren Reactionen wie nach ihrer Formel als eine dem Ornithin homologe Verbindung erschien, das Lysin Drechsels. Zwei Jahre darauf wurde zum ersten

1) Diese Ztschr. Bd. XI, S. 43.

2) Ber. d. deutsch. chem. Ges. 30. 2879 u. diese Ztschr. Bd. XXVI, S. 1.

3) Ber. d. deutsch. chem. Ges. 32, 3191.

4) Ber. d. deutsch. chem. Ges. 10, 1925 u. 11, 406. 
Male eine Diamidosäure der Fettreihe synthetisch erhalten. Klebs ${ }^{1}$ ) stellte in Hüfner's Laboratorium aus $\alpha$ - $p$-Dibrompropionsäure die entsprechende Diamidopropionsäure dar. Sie zeigte sowohl mit dem Ornithin wie mit dem Lysin die grösste Aehnlichkeit in ihrem chemischen Verhalten, so dass die Anschauungen Jaffé's und Drechsel's eine wesentliche Stütze erhielten. Noch aber stand der Beweis für die Richtigkeit ihrer Formeln aus und vor Allem fehlte zur vollen Erkenntniss der Constitution des Ornithins und Lysins die Beantwortung der Frage, welches die Stellung der beiden Amidogruppen sei.

Zwar haben Schulze und Winterstein in der oben erwähnten Arbeit dem Arginin die Constitutionsformel

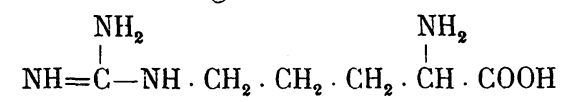

beigelegt, aber sie führen in ihrer ersten Publication für die $u$ - $\delta$-Stellung der beiden Amidogruppen keine Gründe an und in der ausführlicheren Arbeit in dieser Zeitschrift wird höchstens wahrscheinlich gemacht, dass die beiden Amidogruppen sich nicht an benachbarten Kohlenstoffatomen befinden.

Bei den ausserordentlichen Schwierigkeiten, mit welchen die Darstellung einer grösseren Menge von Ornithin verknüpft ist, versprach zur Aufklärung seiner Constitution nur eine solche Methode Erfolg, welche für den einzelnen Versuch nicht zu viel Material erforderte. Frühere Versuche, Spaltungsprodukte des Ornithins zu erhalten, die Herr Professor Jaffé, wie er mir mittheilte, angestellt hatte, hatten wegen Mangels an Material keine entscheidenden Resultate ergeben. Eigene Bemühungen, durch trocknes Erhitzen des salzsauren Ornithins etwa zu einem Pyridin- oder Pyrrolidinderivat zu gelangen, hatten aus dem gleichen Grunde bisher nicht mehr Erfolg. Auch die analogen Versuche Drechsel's ${ }^{2}$ ) mit dem Lysin ermuthigten nicht dazu, äiesen Weg weiter zu betreten. Ich versuchte deshalb nicht mit Hülfe chemischer Agentien, sondern durch die Wirkung von Fäulnissbakterien die Spaltung des Ornithins zu bewirken.

1) Diese Ztschr. Bd. XIX, S. 301.

2) Ber. d. d. chem. Ges. 25, 2454. 
Die Anwendung dieser Methode versprach deshalb von vornherein Erfolg, weil Brieger ${ }^{1}$ ) das Putrescin, welches später durch die Untersuchungen von Ladenburg ${ }^{2}$ ) und Baumann und v. Udranszky ${ }^{3}$ als Tetramethylendiamin identificirt wurde, als Produkt von faulenden, allerdings nicht einheitlichen Eiweisssubstanzen erhalten hatte. Auf die nahen chemischen Beziehungen zwischen dem Ornithin und Putrescin hatten schon Baumann und v. Udranszky aufmerksam gemacht, ohne indessen diesen Gedanken weiter zu verfolgen. Da nun inzwischen das Ornithin als Spaltungsprodukt der Eiweisskörper nachgewiesen worden ist, so eröffnete sich ein neuer Ausblick auf die Entstehungsweise des Putrescins und zugleich die Möglichkeit, die Frage nach der Constitution des Ornithins auf dem angegebenen Wege zu entscheiden.

Es gelang in der That, durch Anwendung der Fäulnissmethode vom Ornithin zum Putrescin zu gelangen und in gleicher Weise lieferte das Lysin das homologe Cadaverin oder Pentamethylendiamin.

\section{Versuche mit dem salzsauren Ornithin.}

Ueber die Darstellung der Ornithursäure aus Hühnerexcrementen füge ich der Beschreibung in der Arbeit von Jaffé einige Erfahrungen hinzu, die im Laufe der Jahre bei der Gewinnung jener Substanz im hiesigen Institute gesammelt worden sind.

Es empfiehlt sich, den Hühnern den Dickdarm oberhalb der Cloake zu unterbinden, um den Urin ohne Kothbeimengung zu erhalten. Die Ausbeuten werden dadurch wesentlich verbessert. Allerdings gehen die Versuchsthiere früher zu Grunde. Immerhin hielten einzelne Thiere die Fütterung von je $0,5 \mathrm{~g}$ benzoesaurem Natron 12-14 Tage lang aus; die durchschnittliche Lebensdauer betrug bei dieser Dosis 7-9 Tage. Grössere Dosen anzuwenden, ist nicht gerathen, obwohl die Thiere die-

1) Die Ptomaine. Berlin 1886.

2) Ber. d. d. chem. Ges. 16, 360.

3) Diese Ztschr. Bd. XIII, S. 562.

Hoppe-Seyler's Zeitschrift f. physiol. Chemie. XXIX. 
selben vertragen, da auch so schon beträchtliche Mengen unveränderter Benzoesäure ausgeschieden werden.

Bei der Reinigung der harzig ausgeschiedenen Ornithursäure ist besonders darauf zu achten, dass beim Uebersättigen des mit Kaliumpermanganat entfärbten Filtrats mit Salzsäure in sehr verdünnter Lösung und möglichst bei niederer Temperatur gearbeitet wird. Nur dann geht die harzige Masse, die meist bald hart und spröde wird, auch in kurzer Zeit in den krystallinischen Zustand über. Bis die letzten Antheile des Harzes krystallinisch geworden sind, vergehen oft mehrere Wochen.

Was die Ausbeuten anlangt, so wurden in einem Falle aus den Excrementen eines Huhnes mit unterbundenem Darm nach Verabreichung von $4 \mathrm{~g}$ benzoesaurem Natron in Dosen von $0,5 \mathrm{~g}$ erhalten: aus der ätherischen Lösung $0,17 \mathrm{~g}$, aus der wässerigen Lösung als Harz 0,95 g (nach Abpressen mit Fliesspapier und Trocknen an der Luft gewogen). An reiner krystallinischer Substanz erhält man etwa die Hälfte von dem Gewicht des Harzes.

Nach einer mehrwöchentlichen Fütterung einer grösseren Zahl von Hühnern mit $205 \mathrm{~g}$ benzoesaurem Natron wurden im Ganzen ca. $33 \mathrm{~g}$ reine Ornithursäure erhalten.

Aus der krystallinischen Ornithursäure wurde durch Kochen mit concentrirter Salzsäure am Rückflusskühler nach der Vorschrift Jaffe's das salzsaure Ornithin dargestellt. Wenn der syrupöse Rückstand nach mehrmaligem Abdampfen der überschüssigen Salzsäure beim Versetzen mit kaltem absoluten Alkohol nicht ohne Weiteres krystallinisch erstarrt, so gelingt es oft, durch Zusatz weniger Tropfen verdünnten Ammoniaks die Krystallisation zu bewirken.

$\mathrm{Zu}$ den Fäulnissversuchen wurde manchmal nicht das krystallisirte Salz benutzt, sondern der nach Verjagen der überschüssigen Salzsäure zurückbleibende Syrup.

Bei den ersten Fäulnissversuchen war die Versuchsanordnung die folgende: Das salzsaure Salz wurde in etwa der hundertfachen Menge Leitungswasser gelöst, mit wenigen Tropfen Sodalösung schwach alkalisch gemacht und mit 
2-3 Flocken eines faulenden Pankreas und einigen Tropfen Faulflüssigkeit versetzt. Am wirksamsten erwiesen sich die Fäulnissbakterien, wenn das in Stücke zerhackte Pankreas unter ganz schwacher Sodalösung in einem mit Wattebausch verschlossenen geräumigen Kolben an einem mässig warmen Orte etwa 24 Stunden gestanden hatte. Die oben beschriebene Lösung wurde in einem offenen oder mit Wattebausch verschlossenen Kolben im Brutschrank 3 Tage lang bei ca. $30^{\circ}$ stehen gelassen. Sie wurde nach Ablauf dieser Frist mit Essigsäure schwach angesäuert, zum Sieden erhitzt, damit die geringen Quantitäten noch vorhandenen Eiweisses coagulirt würden, und filtrirt. Das Filtrat wurde so lange mit Benzoylchlorid und Natronlauge nach der Baumann-Schotten'schen Methode geschüttelt, bis ein neuer Zusatz von Benzoylchlorid keine neue Ausscheidung mehr bewirkte. Es bildete sich eine unlösliche Benzoylverbindung, die sich zunächst schmierig abschied, aber meist schon nach mehrstündigem Stehen ohne Weiteres krystallinisch wurde. Die ausgeschiedene Verbindung wurde abfiltrirt, in heissem Alkohol gelöst und die alkoholische Lösung in etwa die 20 fache Menge kalten Wassers gegossen, worauf sie sich in schönen Nadeln und Blättchen abschied, welche schon nach einmaligem Umkrystallisiren aus Alkohol den Schmelzpunkt $176^{\circ}$ zeigten. Bei mehrmaligem Umkrystallisiren stieg der Schmelzpunkt auf 1780. In der Litteratur finden sich für den Schmelzpunkt des Dibenzoyltetramethylendiamins von verschiedenen Beobachtern beide Angaben.

Die Elementaranalyse ergab für diesen Körper stimmende Zahlen :

I. $0,203 \check{~} \mathrm{~g}$ Substanz gaben im offenen Rohr verbrannt $0,5474 \mathrm{~g}$ $\mathrm{CO}_{2}=73,36 \% \mathrm{C}$ und $0,1256 \mathrm{~g} \mathrm{H}_{2} \mathrm{O}=6,88 \% \mathrm{H}$.

II. $0,2229 \mathrm{~g}$ Substanz gaben nach Dumas $18,6 \mathrm{ccm}$. $\mathrm{N}$ bei $15^{\circ}$ und $762 \mathrm{~mm}$. Druck $=9,80 \% \mathrm{~N}$.

$$
\begin{array}{lllll}
\mathrm{C}_{4} \mathrm{H}_{8}\left(\mathrm{NH} \cdot \mathrm{COC}_{6} \mathrm{H}_{5}\right)_{2} & \begin{array}{l}
\text { Berechnet: } \\
\text { Gefunden: }
\end{array} & \text { C } 72,97 & \text { H 6,75 } & \text { N 9,46 } \\
& \text { G } 6,88 & \text { N 9,80. }
\end{array}
$$

Um die Dibenzoylverbindung vollständig aus der Lösung zu gewinnen, wurde das alkalische Filtrat angesäuert. Der ausgeschiedene Niederschlag wurde abfiltrirt, die saure Lösung 
noch mehrmals ausgeäthert, der Aether fast vollständig abdestillirt und der Rest der ätherischen Lösung in Natronlauge eingetragen. Diese weitere Behandlung, welche Baumann gelegentlich der Isolirung der Diamine aus dem Harn bei Cystinurie empfohlen hat, erwies sich in dem vorliegenden Falle als überflüssig; denn die nachträgliche Ausscheidung der Benzoylverbindung war minimal. Der aus der angesäuerten Lösung nach längerem Stehen ausgeschiedene Niederschlag wurde mehrmals mit Petroläther ausgekocht und heiss filtrirt. Aus dem Rückstande konnte durch Umkrystallisiren aus Alkohol unveränderte reine Ornithursäure gewonnen werden.

Bei einem Versuche wurde ein Theil der Lösung nach dem Faulen nach der Methode Brieger's behandelt, welche er zur Isolirung seiner Ptomaine benutzte. Das Fäulnissprodukt wurde mit Salzsäure schwach angesäuert, auf dem Wasserbade zur Trockne verdampft und der Rückstand mehrfach mit absolutem Alkohol ausgezogen. Die alkoholische Lösung wurde mit alkoholischem Quecksilberchlorid versetzt. Dabei schied sich ein reichlicher Niederschlag aus, welcher abfiltrirt und in wenig Wasser gelöst wurde. Die Lösung wurde mittelst Schwefelwasserstoff vom Quecksilber befreit, filtrirt und zur Trockne verdampft. Der krystallinische Rückstand wurde mit wenig 96\% igem Alkohol warm extrahirt und der in Alkohol unlösliche Theil in wenig Wasser gelöst und unter Zusatz von Alkohol mit wässerigem Platinchlorid gefällt.

Das so erhaltene Platinsalz stimmte in seinen Eigenschaften mit denjenigen des Putrescin-Platinchlorids überein. Die Analyse ergab folgende Zahlen:

0,0820 g Substanz lieferten $0,0320 \mathrm{~g} \mathrm{Pt}=39,03 \%$.

$\mathrm{C}_{4} \mathrm{H}_{8}\left(\mathrm{NH}_{2}, \mathrm{HCl}\right)_{2} \cdot \mathrm{PtCl}_{4} \quad$ Berechnet: Pt 39,15\%. Gefunden: 39,03\%.

Bei den nach der beschriebenen Methode - unter Luftzutritt - angestellten Fäulnissversuchen war die Ausbeute in allen Fällen gering. Mehr als etwa $12 \%$ der theoretischen Menge wurden nie erhalten, in einigen Fällen überhaupt nur Spuren der Benzoylverbindung isolirt. Durch Kontrollversuche wurde ermittelt, dass aus den minimalen Mengen des zugesetzten Pankreas oder der Faulflüssigkeit das gefundene 
Dibenzoylputrescin nicht stammen konnte. Solche Proben ergaben, in der gleichen Weise behandelt, nur eine schwache milchige Trübung, aus welcher sich nach mehrtägigem Stehen in der Kälte manchmal ganz wenige Krystalle abschieden. Nur in einem Versuche war es möglich, ihren Schmelzpunkt zu bestimmen. Derselbe lag nahe dem des Dibenzoylcadaverins. Ob diese Substanz vorlag, liess sich der sehr geringen Menge halber nicht bestimmen. Nach den in der Litteratur vorliegenden Angaben von $\mathrm{Garcia}^{1}$ ) und $\mathrm{Werig0^{2 }}$ ) ist es nicht unwahrscheinlich.

Wesentlich besser wurden die Ausbeuten an Putrescin, wenn bei der Fäulniss der Luftzutritt gänzlich ausgeschlossen wurde. Die Versuchsanordnung, welche bei den späteren Versuchen stets angewandt wurde, war die folgende: das salzsaure Salz wurde in etwa der hundertfachen Menge ausgekochten Leitungswassers gelöst, welchem geringe Mengen von Chlorkalium, Magnesiumsulfat und Natriumphosphat zugesetzt waren, und mit Sodalösung schwach alkalisch gemacht. Die Lösung wurde wie in den früheren Versuchen mit Pankreasflocken und Faulflüssigkeit versetzt und in ein mit doppelt durchbohrtem Kautschukstopfen verschlossenes Kölbchen gebracht, welches sie fast ganz anfüllte. Durch die eine Oeffnung des Stopfens führte ein dünnes Gaszuleitungsrohr bis auf den Boden des Kölbchens; dieses Rohr trug oben ein Stück Gummischlauch, welches durch einen Quetschhahn verschlossen werden konnte. Durch die andere Oeffnung ging ein Glasrohr, das dicht unter dem Stopfen endete und dessen aus dem Stopfen herausragender Theil nach unten umgebogen in Wasser endete. Ueber die unter das Wasser mündende Oeffnung konnte ein Reagenzgläschen zum Auffangen entweichender Gase umgestülpt werden. Durch den Kolben wurde vor dem Versuch mindestens eine halbe Stunde Wasserstoff geleitet. Dann wurde der Quetschhahn verschlossen und die von der Luft gänzlich abgeschlossene Flüssigkeit 3-4 Tage im Brutschrank bei $30-35^{\circ}$ belassen. Die Verarbeitung geschah wie bei den Versuchen mit Luftzutritt.

1) Diese Zeitschr. Bd. XVII, S. 543.

2) Pflüger's Archiv. Bd. 51, S. 352. 
Ein quantitativ durchgeführter Versuch mit dieser Versuchsanordnung ergab folgende Zahlenverhältnisse: Aus 0,74 g krystallinischen salzsauren Ornithins wurden etwas über $0,5 \mathrm{~g}$ reines krystallinisches Dibenzoylputrescin erhalten. 0,278 g Ornithursäure wurden aus der gefaulten Lösung zurückgewonnen entsprechend $0,162 \mathrm{~g}$ salzsaurem Ornithin $\left(1^{1 / 2} \mathrm{HCl}\right)$. Es waren also $0,58 \mathrm{~g}$ des Salzes zersetzt. Die theoretische Ausbeute an Dibenzoylputrescin berechnet sich zu $0,92 \mathrm{~g}$, so dass also $50-60 \%$ der theoretischen Menge erhalten wurden.

Auch auf andere stickstoffhaltige Zersetzungsprodukte des Ornithins wurde in den gefaulten Lösungen gefahndet, insbesondere auf $d$-Amidovaleriansäure, welche unter den Fäulnissprodukten des Eiweisses gelegentlich von E. und H. Salkowski ${ }^{1}$ ) aufgefunden worden ist. Ausser Ammoniak konnte aber keine stickstoffhaltige Substanz isolirt werden.

\section{Versuche mit salzsaurem Lysin.}

Von dem salzsauren Lysin kamen eine Reihe Präparate verschiedener Herkunft und verschiedener Herstellungsweise zur Verwendung. Die ersten Versuche wurden mit Lysin angestellt, welches aus Casein gewonnen war. Ein Theil desselben wurde durch Zersetzung des vorher analysirten Platinsalzes erhalten, ein anderer nach der vortrefflichen Methode Kossel's ${ }^{2}$ ) zunächst als pikrinsaures Salz isolirt und aus diesem in das salzsaure Salz übergeführt. Zahlreiche Fäulnissversuche mit solchem aus Casein stammenden Lysin führten zunächst nicht zu dem gewünschten Ziel. Diese ersten Versuche wurden allerdings zu einer Zeit angestellt, als ich üher die besten Bedingungen der Fäulniss noch nicht viel Erfahrung hatte. Auch überzeugte ich mich dabei noch nicht durch jedesmalige Kontrolle mit Ornithinlösung, ob die zugesetzte Faulflüssigkeit im Stande war, dieses in Putrescin überzuführen, was bei den späteren Versuchen niemals unterblieb, trotzdem ich von dem werthvollen Ornithinsalz auf diese Weise viel opfern musste.

1) Ber. d. d. chem. Gesellsch. Bd. XVI, S. 1191.

2) Diese Zeitschr. Bd. XXVI, S. 586. 
Eine solche Kontrolle halte ich aber jetzt für durchaus nothwendig, so lange man nicht etwa mit Reinculturen von Bakterien arbeitet, von welchen erwiesen ist, dass sie im Stande sind, die Kohlensäureabspaltung aus den Diamidosäuren zu bewirken.

Ich schob nach den verschiedenen Fehlversuchen mit dem Lysin aus Casein die Schuld zunächst auf das Ausgangsmaterial und stellte mir, um die Frage zu entscheiden, ob nur dieses Lysin kein Pentamethylendiamin lieferte, zunächst nach Kutscher's ${ }^{1}$ ) Vorschrift Lysin durch Selbstverdauung von Pankreas dar. Nach den quantitativen Versuchen von Garcia ${ }^{2}$ ) war bekannt, dass bei der Fäulniss des Pankreas reichliche Mengen von Cadaverin auftraten, deren Entstehung aus Lysin ich nach meinen Ornithinversuchen annahm.

Während ich bei einer Darstellung mich streng an die Vorschriften Kutscher's hielt und sogenanntes Trockenpankreas einer mehrmonatlichen Selbstverdauung überliess, kochte ich ein anderes Mal die frische Pankreasdrüse mit Salzsäure und Zinnchlorür nach dem Vorgange von Hlasiwetz und Habermann. ${ }^{3}$ ) Nach meinen quantitativ durchgeführten Versuchen sind dabei die Mengenverhältnisse der entstehenden Basen ungefähr die gleichen wie bei der Selbstverdauung des Pankreas. Die Säurespaltung dürfte sich darum wegen der grossen Zeitersparniss zur Darstellung der Basen mehr empfehlen.

Ein Vergleich des Schmelzpunkts und des Drehungsvermögens der aus Casein und aus Pankreas gewonnenen Hydrochloride des Lysins zeigte, dass aus beiden Materialien dieselben Salze entstanden, und bei den späteren Fäulnissversuchen gelang es denn auch, aus beiden Cadaverin zu isoliren. In dem ersten unter Luftabschluss durchgeführten Versuch war die Ausbeute aus dem vom Pankreas stammenden Präparat grösser, in dem zweiten lieferte dieses nur geringe Mengen, während das Lysin aus Casein eine gute Ausbeute an Cadaverin gab. So grosse Mengen des Diamins wie aus Ornithin

1) Die Endprodukte der Trypsin-Verdauung, Strassburg, 1899.

2) Diese Zeitschr. Bd. XVII, S. 543.

3) Liebig's Ann. d. Chem. 169, 150. 
konnte ich aus Lysin nie erhalten. Ueberhaupt scheint die Fäulniss des Lysins sich schwieriger und langsamer $\mathrm{zu}$ vollziehen.

Die Behandlung des gefaulten Lysins geschah nach derselben Methode, wie sie beim Ornithin beschrieben ist. Bei dem ersten erfolgreichen Versuche geschah die Isolirung nach Baumann-Schotten. Die in Alkohol gelöste, in Wasser gegossene Benzoylverbindung zeigte den Schmelzpunkt 126 - 128". Die Krystalle konnten, der geringen Menge wegen, aus Alkohol nicht umkrystallisirt werden. Sie wurden vielmehr in wenig Alkohol gelöst und mit Wasser bis zur beginnenden Trübung versetzt, die trübe Flüssigkeit wurde durch Erhitzen wieder klar und beim Erkalten schieden sich wohl ausgebildete Blättchen aus, deren Schmelzpunkt zwischen 129-1310 lag. Baumann gibt für das Dibenzoylpentamethylendiamin den Schmelzpunkt $130^{\circ}$ an. Die Analyse stimmte auf diesen Körper:

$0,0987 \mathrm{~g}$ Substanz geben nach Dum as 7,9 ccm. N bei 120 und $761 \mathrm{~mm}$. Druck. $\mathrm{C}_{5} \mathrm{H}_{10}\left(\mathrm{NHCOC}_{6} \mathrm{H}_{5}\right)_{2}$ Ber.: N $9.03 \%$. Gef.: N 9,41\%.

Bei diesem Versuche lieferten $0,477 \mathrm{~g}$ salzsaures Lysin $0,24 \mathrm{~g}$ der krystallinischen, noch nicht umkrystallisirten DibenzoylVerbindung; das entspricht ca. 0,08 Cadaverin oder 36\% der theoretischen Ausbeute.

In einem zweiten Versuche wurde die Lösung nach Brieger's Verfahren verarbeitet. Bei der mehrmaligen Extraction mit 96\% \% igem und mit absolutem Alkohol blieben ansehnliche Mengen von Salmiak zurück. Die alkoholische Lösung wurde mit gesättigter alkoholischer Sublimatlösung gefällt. Aus $1,57 \mathrm{~g}$ salzsaurem Lysin wurden ca. $2 \mathrm{~g}$ des Quecksilber-Doppelsalzes erhalten, welchem nach Brieger und Bocklisch ${ }^{1}$ ) die Formel $\mathrm{C}_{5} \mathrm{H}_{14} \mathrm{~N}_{2} \cdot 2 \mathrm{HCl}, 4 \mathrm{HgCl}_{2}$ zukommt. Das entspricht $0,182 \mathrm{~g}$ Cadaverin oder $25 \%$ der theoretischen Ausbeute.

Das Quecksilbersalz wurde in Wasser gelöst und durch Schwefelwasserstoff zersetzt. Die vom Schwefelquecksilber abfiltrirte Lösung wurde zur Trockne eingedampft; der farblose, krystallinische Rückstand löste sich bei dreimaligem Extrahiren

1) Brieger, die Ptomaine, S. 100. 
vollständig in Alkohol. Auf Zusatz von 20\% Platinchlorid fiel sofort ein krystallinisches Platinsalz aus der alkoholischen Lösung, welches, aus wenig heissem Wasser umkrystallisirt, sich mit dem von Brieger erhaltenen Cadaverinplatinchlorid identisch erwies.

0,2043 g Substanz (bei $100^{\circ}$ getrocknet) lieferten 0,0772 g Platin $\mathrm{C}_{5} \mathrm{H}_{10}\left(\mathrm{NH}_{2}, \mathrm{HCl}\right)_{2} \mathrm{PtCl}_{4} \quad$ Ber.: Pt 38,02\% Gef.: Pt 38,28\%.

Mit alkoholischer oder wässeriger Pikrinsäure gibt die alkoholische Lösung des salzsauren Salzes ein in Alkohol und Wasser schwer lösliches Pikrat vom Schmelzpunkt 221-2230. Brieger gibt $222^{\circ}$ an. Auch die übrigen für das Cadaverin angegebenen Reactionen gegenüber Alkaloidreagentien wurden mit der aus Lysin gewonnenen Base erhalten, so dass an ihrer Identität mit Cadaverin kein Zweifel sein kann. Davon, dass das angewandte Lysin nicht etwa mit Cadaverin von vornherein verunreinigt war, überzeugte ich mich durch Benzoylirung nach Schotten-Baumann. Aus der alkalischen Lösung schied sich dabei keine Benzoylverbindung ab.

\section{Besprechung der Versuchsergebnisse.}

Durch den Uebergang von Ornithin in Putrescin bei der Fäulniss ist erwiesen, dass den beiden Amidogruppen im Ornithin die $\alpha$ - $\delta$-Stellung zukommt, welche für das Putrescin von Ladenburg ${ }^{1}$ ) durch die Gewinnung des Putrescins aus Aethylencyanid auf dem Wege der Reduction erwiesen ist. Eine Atomumlagerung anzunehmen, liegt kein Grund vor, zumal der Process relativ glatt verläuft, wie die Ausbeute von $50-60 \%$ Putrescin unter günstigen Bedingungen beweist. Ebenso wenig ist ein Grund zu der Annahme vorhanden, dass etwa die Fäulnissbakterien das Ornithin nur als ein geeignetes Nährmaterial benutzen und das Putrescin als ein von dieser Nahrung unabhängiges Stoffwechselprodukt ausscheiden. Sonst wäre nicht einzusehen, warum sie gerade nach OrnithinAufnahme Putrescin, nach Lysin-Nahrung Cadaverin ausscheiden.

1) Ber. d. d. chem. Ges. 1. c. 
Man kann also den Process der Putrescinbildung durch die folgende Gleichung ausdrücken:

$$
\begin{gathered}
\mathrm{CH}_{2}\left(\mathrm{NH}_{2}\right) \cdot \mathrm{CH}_{2} \cdot \mathrm{CH}_{2} \cdot \mathrm{CH}\left(\mathrm{NH}_{2}\right) \cdot \mathrm{COOH}=\mathrm{CO}_{2}+ \\
\mathrm{CH}_{2}\left(\mathrm{NH}_{2}\right) \cdot \mathrm{CH}_{2} \cdot \mathrm{CH}_{2} \cdot \mathrm{CH}_{2}\left(\mathrm{NH}_{2}\right) \cdot
\end{gathered}
$$

Nicht streng erwiesen ist allerdings bisher, dass eine Amidogruppe mit dem Carboxyl an demselben Kohlenstoffatom haftet. Aber es ist gewiss die wahrscheinlichste Annahme, wenn man bedenkt, dass die Monoamidosäuren, die als Spaltungsprodukte aus Eiweisskörpern künstlich erhalten worden sind oder im Thierkörper vorkommen, fast sämmtlich diese Atomgruppirung zeigen, so das Glycocoll, Alanin, die $"$-Amidovaleriansäure, das Leucin und Tyrosin. Noch ein Weiteres spricht dafür: Die bereits angeführte $\delta$-Amidovaleriansäure, welche E. und H. Salkowski bei der Fäulniss von Fleisch auffanden und Gabriel ${ }^{1}$ ) mit seiner synthetisch erhaltenen Säure identificirte, darf wohl ebenfalls als ein Abkömmling des Ornithins aufgefasst werden, obwohl ich sie bisher nicht bei der Fäulniss dieses Körpers nachweisen konnte. Ist diese Voraussetzung richtig, so ist damit auch die Stellung der fraglichen Amidogruppe zum Carboxyl festgesetzt, da über die Stellung der beiden Amidogruppen zu einander kein Zweifel möglich ist.

Nehmen wir also für das Ornithin die obige Formel an, so ist damit eine wichtige Erkenntniss für den Weg gewonnen, wie aus dem Eiweiss Pyridinderivate entstehen können, ohne dass wir in dem Eiweissmolekül einen präformirten Pyridinring annehmen müssen. Solche Pyridinderivate, die vielleicht aus Eiweiss entstehen, liefert uns aber nicht nur die Pflanze in zahlreichen Alkaloiden, sondern auch der Thierkörper, wie z. B. die Untersuchungen v. Fürth's ${ }^{2}$ ) über die wirksame Substanz der Nebennieren andeuten. Wenn sich die Ansicht dieses Autors über die Constitution des Suprarenins durch die in Aussicht gestellten weiteren Analysen bestätigt, so ist die Beziehung zwischen dieser Substanz und dem Ornithin eine nahe.

1) Gabriel u. Oschan, Ber. d. d. chem. Ges. 24, 1364 .

2) Diese Ztschr. Bd. XXVI, S. 15. 
v. Fürth spricht dieselbe als ein Tetrahydrodioxypyridin an. Es braucht im Ornithin nur die $\alpha$-Amidogruppe durch Hydroxyl ersetzt $\mathrm{zu}$ werden, damit aus demselben durch Wasseraustritt ein Körper von der Formel des Suprarenins entsteht. Die analoge Bildung eines Oxypiperidins aus $\delta$-Amidovaleriansäure ist bereits durch Schotten ${ }^{1}$ ) erwiesen.

Analoge Betrachtungen wie diejenigen über die Beziehungen des Ornithins zum Putrescin gelten auch für das Verhältniss des Lysins zum Cadaverin. Dasselbe wird durch die Gleichung veranschaulicht :

$\mathrm{CH}_{2}\left(\mathrm{NH}_{2}\right) \cdot \mathrm{CH}_{2} \cdot \mathrm{CH}_{2} \cdot \mathrm{CH}_{2} \cdot \mathrm{CH}\left(\mathrm{NH}_{2}\right) \cdot \mathrm{COOH}=\mathrm{CO}_{2}+$

$\mathrm{CH}_{2}\left(\mathrm{NH}_{2}\right) \cdot \mathrm{CH}_{2} \cdot \mathrm{CH}_{2} \cdot \mathrm{CH}_{2} \cdot \mathrm{CH}_{2} \cdot \mathrm{NH}_{2}$.

Auch auf dem Wege über das Lysin ist somit ein Uebergang von den Eiweisskörpern zum Pyridinring gegeben. Denn wie Ladenburg' ${ }^{2}$ ) gezeigt hat, bildet sich aus dem Pentamethylendiamin durch trockenes Erhitzen unter AmmoniakAbspaltung Piperidin.

Durch die beschriebenen Versuche findet aber auch zugleich die Entstehung der beiden bei der Eiweissfäulniss in grösster Menge entstehenden Basen Brieger's eine einfache Erklärung. Baumann hatte noch angenommen, dass diese Basen synthetisch aus 2 Molekülen Monaminen, deren Entstehung bei der Eiweissfäulniss von Brieger, Nencki u. A. beobachtet worden ist, unter Mitwirkung von Sauerstoff gebildet wurden. Er drückte die beiden Vorgänge durch die Gleichungen aus :

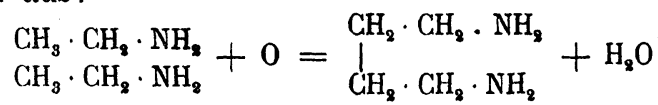

$$
\begin{aligned}
& \begin{array}{l}
\text { und } \mathrm{CH}_{3} \cdot \mathrm{CH}_{2} \cdot \mathrm{NH}_{2} \\
\mathrm{CH}_{3} \cdot \mathrm{CH}_{2} \cdot \mathrm{CH}_{2} \cdot \mathrm{NH}_{2}
\end{array}
\end{aligned}
$$

Diese Auffassung, welche auch in die Lehrbücher der physiologischen Chemie übergegangen ist, scheint mir schon deshalb unhaltbar, weil die Ausbeute an den Diaminen gerade bei gänzlichem Sauerstoffmangel die bedeutendste ist. An ihre Stelle wird vielmehr die treten müssen, dass durch hydrolytische

1) Ber. d. d. chem. Ges. 21, 2235.

2) Ber. d. d. chem. Ges. 18, 3100 . 
Spaltung aus den Eiweisskörpern Lysin und Arginin und aus diesem weiterhin Ornithin entsteht, und dass die beiden Diamidosäuren unter Kohlensäure-Abspaltung die Diamine liefern.

Nach dieser Annahme darf man erwarten, dass Putrescin in reichlicher Menge bei der Fäulniss solcher Substanzen auftritt, die bei der hydrolytischen Spaltung viel Arginin liefern. Durch Kossel's Untersuchungen über die Protamine aus dem Fischsperma haben wir in dieser Substanz eine solche Argininquelle kennen gelernt. Es gelang mir in der That, aus frischem Fischsperma grosse Quantitäten von Putrescin bei der Fäulniss $\mathrm{zu}$ isoliren.

Durch die Anwendung der Fäulnissmethode ist es gelungen, unsere Kenntnisse über die Constitution des Arginins bezw. Ornithins und Lysins zu einem gewissen Abschlusse zu bringen. Sie dürfte sich in manchen andern Fällen zur Aufklärung von Constitutionsfragen gerade bei solchen Körpern empfehlen, von welchen nur geringe Mengen zur Verfügung stehen.

Königsberg i. Pr., 15. März 1900. 